\title{
Ki-67 is a Valuable Prognostic Factor in Gliomas: Evidence from a Systematic Review and Meta-analysis
}

\author{
Wen-Jie Chen ${ }^{1}$, De-Shen He ${ }^{2}$, Rui-Xue Tang ${ }^{1}$, Fang-Hui Ren ${ }^{1}$, Gang Chen ${ }^{1 *}$
}

\begin{abstract}
Ki-67 has been widely used as an indicator of cell proliferation in gliomas. However, the role of Ki-67 as a prognostic marker is still undefined. Thus, we conducted a meta-analysis of the published literatures in order to clarify the impact of Ki-67 on survival in glioma cases. Eligible studies were identified in PubMed, EMBASE, ISI Web of Science, Cochrane Central Register of Controlled Trials, Science Direct and Wiley Online Library with the last search updated on August 31, 2014. The clinical characteristics, overall survival (OS) and progressionfree survival (PFS) together with Ki-67 expression at different time points were extracted. A total of 51 studies, covering 4,307 patients, were included in the current meta-analysis. The results showed that overexpression of Ki-67 can predict poor OS $(\mathrm{HR}=1.66,95 \% \mathrm{CI}$ : $1.53-1.80 ; \mathrm{Z}=11.87 ; p=0.000)$ and poor PFS $(\mathrm{HR}=1.67,95 \% \mathrm{CI}$ : 1.47-1.91; $Z=7.67 ; p=0.000$ ) in gliomas. Moreover, subgroup analyses also indicated that high level of Ki-67 expression was related to poor OS and PFS in glioma patients regardless of region, pathology type, cut-off value and statistical method. In conclusion, the current meta-analysis revealed that $\mathrm{Ki}$-67 expression might be a predicative factor for poor prognosis of glioma patients, emphasizing its importance as a predictor.
\end{abstract}

Keywords: Ki-67 - glioma - meta-analysis - immunohistochemistry - prognosis - survival

Asian Pac J Cancer Prev, 16 (2), 411-420

\section{Introduction}

Glioma is the most common intracranial neoplasia in adults, which arises from the brain or spinal cord tissues (Hernandez-Pedro et al., 2013). Glioma accounts for approximately $80 \%$ of all primary malignant brain tumors (Kohler et al., 2011). Malignant glioma is the most frequent type of primary brain tumors, with an annual incidence of 5/100, 000 individuals (Wen and Kesari, 2008). The highly invasive nature of this tumor prevents complete tumor resection and causes significant neurologic morbidity and mortality (Kouri et al., 2012; Zhao et al., 2013). Despite advances in diagnostic and therapeutic techniques, the prognosis for most glioma patients remains dismal (Nazarenko et al., 2012; Westermark, 2012).

$\mathrm{Ki}-67$, a non-histone protein, is a DNA-binding nuclear protein expressed throughout the cell cycle in proliferating cells, but not in quiescent (G0) cells (Tadbir et al., 2012; Fakhrjou et al., 2013). Ki-67 has been used to distinguish growing cells from non-growing cells (Haroon et al., 2013; Dang et al., 2014; Huang and Chen, 2014). Furthermore, $\mathrm{Ki}-67$ is a reliable indicator of tumor cell proliferative activity that has been associated with the histological grade for glioma (Hu et al., 2013). In spite of a large number of studies performed in glioma patients, the prognostic value of Ki-67 for survival remains controversial. Therefore, we performed a systematic review of the literatures with meta-analysis to assess the prognostic value of Ki-67 for prognosis.

\section{Materials and Methods}

\section{Literature search}

A literature search via PubMed, EMBASE, ISI Web of Science, Cochrane Central Register of Controlled Trials, Science Direct and Wiley Online Library was conducted to search articles that evaluated Ki-67 in glioma (Last search was updated on August31, 2014). The keywords and text words were used as follows: (1) ("glioma" OR "astrocytoma" OR "glioblastoma" OR "oligodendroglioma" OR "oligoastrocytoma" OR "Ependymomas" OR "Gliomatosis Cerebri” OR "brain cancer" OR "brain neoplasm" OR "brain tumor" OR "GBM" OR “AA" OR “AO” OR "DIPG”); (2) (“Ki-67” OR "Ki67” OR "MIB-1" OR "proliferative index" OR "proliferative activity" OR "mitotic index" OR "labeling index" OR "mitotic count" OR "proliferative marker"); (3) ("prognos*" OR "surviv*" OR "follow-up" OR "mortality" OR "predict” OR “outcome”).

\section{Selection Criteria}

All English and Chinese studies were included and

${ }^{1}$ Department of Pathology, First Affiliated Hospital of Guangxi Medical University, Nanning, ${ }^{2}$ Department of Neurology, Seventh Affiliated Hospital of Guangxi Medical University, Wuzhou Worker Hospital, Wuzhou, Guangxi Zhuang Autonomous Region, China *For correspondence: chen_gang_triones@163.com 
Wen-Jie Chen et al

all eligible articles that examined the association between the expression of Ki-67 and overall survival (OS) or progression free survival (PFS) were gathered. However, the papers which only have abstracts were excluded because of insufficient data for meta-analysis. Hence, we first checked the titles of the publications and the abstracts to find exactly those articles that examined the relationship between Ki-67 and OS or PFS in glioma patients. After the abstracts met these conditions, the full texts were analyzed and included into our meta-analysis according to the following criteria: $(i)$ studies were written as full paper; (ii) expression levels of Ki-67 were compared to OS or PFS; ( iii) expression of the protein was evaluated in tumor tissues by immunohistochemistry (IHC), western blot or mRNA by reverse transcription and polymerase chain reaction (RT-PCR) analysis; (iv) Hazard ratios (HR) and $95 \% \mathrm{CI}$ for OS or PFS were provided or could be calculated from the sufficient data.

\section{Data extraction}

Two reviewers independently reviewed all articles and extracted data in separate databases. The following information was collected from each study: name of first author, year of publication, pathology type, WHO grade, number of patients involved, Ki-67 assay, cut-off value, HR with 95\%CI. Disagreements were resolved through discussion among the authors.

\section{Statistical analysis}

A study was considered as significant if the P-value was 0.05 for the statistical test comparing the survival distributions between the groups of low and high Ki-67 expression. A study was classified as 'positive' when Ki-67 expression was identified as a good prognosis factor for survival in univariate analysis. While a study was regarded as 'negative' if the Ki-67 overexpression was associated with a significant detrimental effect on survival. Finally, a study was considered as 'not significant' if no difference between groups expressing or not $\mathrm{Ki}-67$ was detected. The intensity of relationship between the expression levels of Ki-67 and OS or PFS was described as HRs. Positive expression of $\mathrm{Ki}-67$ indicated poor prognosis in patients with glioma if $\mathrm{HR}>1$ with the 95\%CI not overlapping 1 . From some published researches, HR and 95\%CI could be directly obtained by using univariate or multivariate survival analysis. Otherwise, HR and 95\%CI were calculated by Kaplan-Meier survival curves using the software Engauge Digitizer Version4.1 (http://digitizer. sourceforge.net/) and the method (Parmar et al., 1998). $\mathrm{HR}$ and $95 \% \mathrm{CI}$ were also calculated from the sufficient data by SPSS20.0.The pooled HR corresponding to the $95 \% \mathrm{CI}$ was used to assess the prognostic value of $\mathrm{Ki}-67$ in patients. Statistical heterogeneity was tested by Cochrane's $\mathrm{Q}$ test (Chi-squared test; $\left.\mathrm{Chi}^{2}\right)$ and inconsistency $\left(\mathrm{I}^{2}\right)(\mathrm{Lau}$ et al., 1997; Higgins and Thompson, 2002). If there was no obvious heterogeneity, the fixed-effects model (MantelHaenszel method) was used to estimate the pooled HR. Otherwise the random-effects model (DerSimonian and Laid method) was applied. Funnel plot and Begg's rank correlation method were designed for assessing risk of publication bias. STATA 11.0 (STATA Corp., College,
TX) was used to perform statistical analysis.

\section{Results}

\section{Studies selection and characteristics}

The abstracts and titles of primary 924 studies were identified by using the search strategies. Following deduplication, two reviewers independently screened the identified titles and abstracts. They subsequently agreed that 141 articles should be retrieved for detailed review; for these manuscripts, full texts were obtained. On cautious review of study methodologies, 71 of the 141 studies were excluded for showing no information related to $\mathrm{Ki}-67 /$ MIB 1 and survival. Of the candidate studies, 13 articles failed to completely describe the available survival data. Finally, 51 published studies in total were included in the analysis after eliminating articles unsatisfying the selection criteria and duplicates (Jaros et al., 1992; Torp et al., 1992; Montine et al., 1994; Ellison et al., 1995; Heegaard et al., 1995; Kros et al., 1996; Coons et al., 1997; Pollack et al., 1997; Dehghani et al., 1998; McKeever et al., 1998; Ritter et al., 1998; Figarella-Branger et al., 2000; Rodriguez-Pereira et al., 2000; Ho et al., 2001; ReaveyCantwell et al., 2001; Zhong et al., 2001; Bredel et al., 2002; Pollack et al., 2002; Bowers, 2003; Chiang et al., 2003; Neder et al., 2003; Wessels et al., 2003; Zamecnik et al., 2003; Preusser et al., 2005; Uematsu et al., 2005; Kleinschmidt-DeMasters et al., 2006; Donato et al., 2007; Kanamori et al., 2008; Kuo et al., 2009; Laks et al., 2009; Li et al., 2009; Armstrong et al., 2010; Nabika et al., 2010; Watanabe et al., 2010; Yoshida et al., 2010; Habberstad et al., 2011; Margraf et al., 2011; Qiang et al., 2011; Shen et al., 2011; Zawrocki et al., 2011; Okita et al., 2012; Park et al., 2012; Phi et al., 2012; Preusser et al., 2012; Tove et al., 2012; Abd El Atti et al., 2013; Huang et al., 2013; Liu et al., 2013; Yang et al., 2013; Tian et al., 2014; Yue et al., 2014). A flow diagram of the study selection process was presented in Figure1.

The main information of the 51 articles was summarized in Table1. Considering the selected studies, 17 were carried out in Europe, 15 in America, 18 in Asia and 1 in Africa. Immunohistochemistry (IHC) was the only technique performed to detect the expression of $\mathrm{Ki}$ 67 protein. Out of 51 studies published between the years 1992 and 2014, all had the sufficient information for HR extraction, including 46 studies evaluable for OS and 16 for PFS. In the studies with OS, 16 studies provided the multivariate HRs, 9 articles also reported the univariate HRs, 15 showed survival curves available to calculate the HRs, while 7 studies provided the sufficient data to be calculated by SPSS20.0 and 3 studies showed HRs without mentioning the methods used. In the PFS analysis group, 8 studies reported HRs with $95 \% \mathrm{CI}$ in the multivariate models, 8 studies showed the HRs according to univariate analysis, 5 studies showed survival curves that were available to calculate the HRs and 1 study provided the data that could be calculated by SPSS20.0. Subsequently, the perspective of individual glioma pathology type was taken into account. In the OS analysis group, astrocytoma was studied most with twenty-nine studies being included, while 4 studies included oligodendroglial and 
Ki-67 is a Valuable Prognostic Factor of Glioma: Evidence from a Systematic Review and Meta-analysis Table 1. Characteristics of Studies Included in the Meta-analysis

\begin{tabular}{|c|c|c|c|c|c|c|c|c|c|}
\hline Referenc & year & $\begin{array}{l}\text { Re- } \\
\text { gion }\end{array}$ & $\begin{array}{l}\text { Cancer } \\
\text { type }(\mathrm{N})\end{array}$ & Grade & $\begin{array}{c}\mathrm{N} \\
(\mathrm{M} / \mathrm{F})\end{array}$ & cutoff & OS & PFS & HR \\
\hline Torp & 1992 & $\begin{array}{l}\text { Nor- } \\
\text { way }\end{array}$ & GBM20 & IV & $\begin{array}{l}20 \\
(11 / 9)\end{array}$ & $\begin{array}{l}\mathrm{Ki}-67> \\
1.5 \%\end{array}$ & \multicolumn{2}{|c|}{$p=0.12, \mathrm{HR}=2.27(0.82,6.38)$} & Estimated \\
\hline Jaros & 1992 & UK & $\begin{array}{l}\text { GBM8, } \\
\text { AA32 }\end{array}$ & III-IV & $\begin{array}{l}40 \\
(27 / 13)\end{array}$ & $\begin{array}{l}\mathrm{Ki}-67> \\
5 \%\end{array}$ & \multicolumn{2}{|c|}{$p<0.00, \mathrm{HR}=7.36(2.84,19.09)$} & Estimated \\
\hline Montine & 1994 & US & $\begin{array}{l}\text { A11, } \\
\text { AA12, } \\
\text { GBM13 }\end{array}$ & II-IV & $\begin{array}{l}36 \\
(17 / 19)\end{array}$ & $\begin{array}{l}\text { Ki-67 } \geq \\
7.5 \%:\end{array}$ & $\begin{array}{l}p<0.00, \mathrm{HR}=1.84 \\
(0.48,7.05)\end{array}$ & & $\begin{array}{l}\text { Survival } \\
\text { curve }\end{array}$ \\
\hline \multirow{5}{*}{ Ellison } & \multirow{5}{*}{1995} & \multirow{5}{*}{ UK } & \multirow{5}{*}{$\begin{array}{l}\text { FA24, } \\
\text { AA31, } \\
\text { GBM68 }\end{array}$} & \multirow{5}{*}{ III-IV } & \multirow{5}{*}{\begin{tabular}{|l}
123 \\
$(77 / 46)$
\end{tabular}} & $\begin{array}{l}\text { Ki-67> } \\
2 \%,\end{array}$ & $\begin{array}{l}p=0.04, \mathrm{HR}=1.04 \\
(1.01,1.06)\end{array}$ & & ND \\
\hline & & & & & & $\begin{array}{l}\mathrm{Ki}-67 \\
2-5 \%\end{array}$ & $\begin{array}{l}p<0.00, \mathrm{HR}=3.13 \\
(1.81,5.44)\end{array}$ & & $\begin{array}{l}\text { HR (Un- } \\
\text { adjusted) }\end{array}$ \\
\hline & & & & & & $\begin{array}{l}\text { Ki-67 } \\
2-5 \% \\
\end{array}$ & $\begin{array}{l}p=0.40, \mathrm{HR}=1.43 \\
(0.76,2,66)\end{array}$ & & $\begin{array}{l}\text { HR (Ad- } \\
\text { justed) }\end{array}$ \\
\hline & & & & & & $\begin{array}{l}\mathrm{Ki}-67 \\
>5 \%\end{array}$ & $\begin{array}{l}p<0.00, \mathrm{HR}=3.21 \\
(1.95,5,28)\end{array}$ & & $\begin{array}{l}\text { HR (Un- } \\
\text { adjusted) }\end{array}$ \\
\hline & & & & & & $\begin{array}{l}\mathrm{Ki}-67 \\
>5 \%\end{array}$ & $\begin{array}{l}p=0.40, \mathrm{HR}=1.04 \\
(0.55,1.95)\end{array}$ & & $\begin{array}{l}\text { HR (Ad- } \\
\text { justed) }\end{array}$ \\
\hline $\begin{array}{l}\text { Hee- } \\
\text { gaard }\end{array}$ & 1995 & $\begin{array}{l}\text { Den- } \\
\text { mark }\end{array}$ & ODG28 & II & 28 & $\begin{array}{l}\mathrm{Ki}-67> \\
3 \%\end{array}$ & \multicolumn{2}{|c|}{$p<0.00, \mathrm{HR}=2.97(1.04,8.50)$} & \begin{tabular}{|l} 
Survival \\
curve
\end{tabular} \\
\hline \multirow{2}{*}{ Kros } & \multirow{2}{*}{1996} & \multirow{2}{*}{$\begin{array}{l}\text { Neth- } \\
\text { er- } \\
\text { lands }\end{array}$} & \multirow{2}{*}{$\begin{array}{l}\text { ODG } \\
108\end{array}$} & \multirow{2}{*}{ II } & \multirow{2}{*}{108} & $\begin{array}{l}\text { MIB- } \\
1>10 \%\end{array}$ & $\begin{array}{l}p=0.001, \mathrm{HR}=1.59 \\
(1.06,2.40)\end{array}$ & & \begin{tabular}{|l} 
Survival \\
curve
\end{tabular} \\
\hline & & & & & & $\begin{array}{l}\text { MIB- } \\
1 \geq 20 \%\end{array}$ & $\begin{array}{l}p=0.01, \mathrm{HR}=1.73 \\
(1.13,2.65)\end{array}$ & & \begin{tabular}{|l} 
Survival \\
curve
\end{tabular} \\
\hline Pollack & 1997 & US & $\begin{array}{l}\text { GBM16, } \\
\text { AA10 }\end{array}$ & III-IV & \begin{tabular}{|l}
26 \\
$(12 / 14)$ \\
\end{tabular} & $\begin{array}{l}\text { MIB- } \\
1>12 \%\end{array}$ & $\begin{array}{l}p=0.04, \mathrm{HR}=3.88 \\
(1.09,13.8)\end{array}$ & $\begin{array}{l}p=0.04, \mathrm{HR}=3.19 \\
(1.03,9.87)\end{array}$ & Estimated \\
\hline Coons & 1997 & US & $\begin{array}{l}\text { ODG55, } \\
\text { OA26 }\end{array}$ & II & \begin{tabular}{|l|}
81 \\
$(51 / 30)$
\end{tabular} & $\begin{array}{l}\mathrm{Ki}-67> \\
5 \%\end{array}$ & \multicolumn{2}{|c|}{$p=0.04, \mathrm{HR}=5.86(2.65,12.95)$} & \begin{tabular}{|l} 
Survival \\
curve
\end{tabular} \\
\hline Ritter & 1998 & US & E34 & II & \begin{tabular}{|l|}
34 \\
$(19 / 15)$ \\
\end{tabular} & $\begin{array}{l}\text { MIB- } \\
1>20 \%\end{array}$ & $\begin{array}{l}p=0.00, \mathrm{HR}=5.7 \\
(2.00,16.4)\end{array}$ & & ND \\
\hline McKeever & 1998 & US & A50 & II & \begin{tabular}{|l}
50 \\
$(35 / 15)$ \\
\end{tabular} & $\begin{array}{l}\text { MIB- } \\
1>2 \%\end{array}$ & $p=0.00, \mathrm{HR}=1.301$ & $.16,1.46)$ & $\begin{array}{l}\text { HR (Mul- } \\
\text { tivariate) }\end{array}$ \\
\hline \multirow{2}{*}{$\begin{array}{l}\text { Deh- } \\
\text { ghani }\end{array}$} & \multirow{2}{*}{1998} & \multirow{2}{*}{$\begin{array}{l}\text { Ger- } \\
\text { many }\end{array}$} & \multirow{2}{*}{ ODG82 } & \multirow{2}{*}{ II } & \multirow{2}{*}{82} & $\begin{array}{l}\mathrm{Ki}-67> \\
2 \%\end{array}$ & $\begin{array}{l}p<0.00, \mathrm{HR}=7.60 \\
(2.97,19.4)\end{array}$ & & \begin{tabular}{|l} 
Survival \\
curve
\end{tabular} \\
\hline & & & & & & $\begin{array}{l}\mathrm{Ki}-67> \\
5 \%\end{array}$ & $\begin{array}{l}p=0.03, \mathrm{HR}=1.88 \\
(0.94,3.74)\end{array}$ & & \begin{tabular}{|l} 
Survival \\
curve
\end{tabular} \\
\hline $\begin{array}{l}\text { Fiarella- } \\
\text { Branger }\end{array}$ & 2000 & US & E37 & II & \begin{tabular}{|l}
37 \\
$(21 / 16)$ \\
\end{tabular} & $\begin{array}{l}\mathrm{Ki}-67 \\
>1 \%\end{array}$ & $\begin{array}{l}p=0.01, \mathrm{HR}=3.18 \\
(1.40,7.22)\end{array}$ & $\begin{array}{l}p=0.00, \mathrm{HR}=3.18 \\
(1.40,7.22)\end{array}$ & $\begin{array}{l}\text { HR (Mul- } \\
\text { tivariate) }\end{array}$ \\
\hline $\begin{array}{l}\text { Rodri- } \\
\text { guez- } \\
\text { Pereira }\end{array}$ & 2000 & Spain & $\begin{array}{l}\text { A42, } \\
\text { AA25, } \\
\text { GBM25, } \\
\text { ODG62, } \\
\text { AODG, } \\
\text { OA3 }\end{array}$ & II-IV & $\begin{array}{l}137 \\
(85 / 52)\end{array}$ & $\begin{array}{l}\text { MIB-1 in } \\
1 \%\end{array}$ & \multicolumn{2}{|c|}{$p<0.00, \mathrm{HR}=1.03(1.02,1.07)$} & $\begin{array}{l}\text { HR (Mul- } \\
\text { tivariate) }\end{array}$ \\
\hline \multirow{2}{*}{$\begin{array}{l}\text { Reavey- } \\
\text { Cantwell }\end{array}$} & \multirow{2}{*}{2001} & \multirow{2}{*}{ US } & \multirow{2}{*}{ GBM32 } & \multirow{2}{*}{ IV } & \multirow{2}{*}{$\begin{array}{l}32 \\
(22 / 10)\end{array}$} & \multirow{2}{*}{$\begin{array}{l}\text { Ki-67> } \\
20 \%\end{array}$} & $\begin{array}{l}p=0.16, \mathrm{HR}=1.8 \\
(0.8,4.2)\end{array}$ & & $\begin{array}{l}\text { HR(Uni- } \\
\text { variate) }\end{array}$ \\
\hline & & & & & & & $\begin{array}{l}p=0.09, \mathrm{HR}=2.15 \\
(0.89,5.17)\end{array}$ & & $\begin{array}{l}\text { HR (Mul- } \\
\text { tivariate) }\end{array}$ \\
\hline Zhong & 2001 & China & A94 & ND & 94 & $\begin{array}{l}\text { Ki- } \\
67>8.5 \% \\
\end{array}$ & \multicolumn{2}{|c|}{$p=0.00, \mathrm{HR}=6.88(2.75,10.2)$} & \begin{tabular}{|l} 
Survival \\
curve
\end{tabular} \\
\hline Но & 2001 & $\begin{array}{l}\text { Tai- } \\
\text { wan }\end{array}$ & $\begin{array}{l}\text { E48, } \\
\text { AE24, } \\
\text { ME9 }\end{array}$ & II-III & 81 & $\begin{array}{l}\text { MIB- } \\
1 \geq 6 \%\end{array}$ & $\begin{array}{l}p=0.01, \mathrm{HR}=16.5 \\
(2.1,127.2)\end{array}$ & $\begin{array}{l}p<0.00, \mathrm{HR}=23.5 \\
(5.4,101.8)\end{array}$ & $\begin{array}{l}\text { HR (Uni- } \\
\text { variate) }\end{array}$ \\
\hline Bredel & 2002 & $\begin{array}{l}\text { Aus- } \\
\text { tria }\end{array}$ & $\begin{array}{l}\text { GBM } \\
111\end{array}$ & IV & 111 & $\begin{array}{l}\text { MIB- } \\
1 \geq 27 \%\end{array}$ & $\begin{array}{l}p=0.04, \mathrm{HR}=1.49 \\
(1.03,2.14)\end{array}$ & & \begin{tabular}{|l} 
Survival \\
curve
\end{tabular} \\
\hline
\end{tabular}


Wen-Jie Chen et al

\begin{tabular}{|c|c|c|c|c|c|c|c|c|c|}
\hline \multirow{6}{*}{ Pollack } & \multirow{6}{*}{2002} & \multirow{6}{*}{ US } & \multirow{2}{*}{ AA43 } & \multirow{2}{*}{ III } & \multirow{6}{*}{98} & $\begin{array}{l}\text { MIB- } \\
1>18 \%\end{array}$ & & $\begin{array}{l}p=0.02, \mathrm{HR}=2.49 \\
(1.17,5.32)\end{array}$ & $\begin{array}{l}\text { Survival } \\
\text { curve }\end{array}$ \\
\hline & & & & & & $\begin{array}{l}\text { MIB- } \\
1>36 \%\end{array}$ & & $\begin{array}{l}p=0.02, \mathrm{HR}=5.86 \\
(2.71,12.7)\end{array}$ & $\begin{array}{l}\text { Survival } \\
\text { curve }\end{array}$ \\
\hline & & & \multirow{2}{*}{ GBM 42} & \multirow{2}{*}{ IV } & & $\begin{array}{l}\text { MIB- } \\
1>18 \%\end{array}$ & & $\begin{array}{l}p=0.04, \mathrm{HR}=1.69 \\
(1.01,2.85)\end{array}$ & $\begin{array}{l}\text { Survival } \\
\text { curve }\end{array}$ \\
\hline & & & & & & $\begin{array}{l}\text { MIB- } \\
1>36 \%\end{array}$ & & $\begin{array}{l}p=0.04, \mathrm{HR}=2.33 \\
(1.23,4.41)\end{array}$ & $\begin{array}{l}\text { Survival } \\
\text { curve }\end{array}$ \\
\hline & & & \multirow{2}{*}{$\begin{array}{l}\text { Other } \\
\text { eligible13 }\end{array}$} & \multirow{2}{*}{ ND } & & $\begin{array}{l}\text { MIB- } \\
1>18 \%\end{array}$ & & $\begin{array}{l}p<0.01, \mathrm{HR}=1.71 \\
(1.05,2.79)\end{array}$ & $\begin{array}{l}\text { Survival } \\
\text { curve }\end{array}$ \\
\hline & & & & & & $\begin{array}{l}\text { MIB- } \\
1>36 \%\end{array}$ & & $\begin{array}{l}p<0.01, \mathrm{HR}=2.09 \\
(1.32,3.31)\end{array}$ & $\begin{array}{l}\text { Survival } \\
\text { curve }\end{array}$ \\
\hline \multirow{2}{*}{ Wessels } & \multirow{2}{*}{2003} & \multirow{2}{*}{ UK } & \multirow{2}{*}{ A47 } & \multirow{2}{*}{ II } & \multirow{2}{*}{$\begin{array}{l}47 \\
(25 / 22)\end{array}$} & \multirow{2}{*}{$\begin{array}{l}\text { MIB- } \\
1>1 \%\end{array}$} & $\begin{array}{l}p=0.02, \mathrm{HR}=3.63 \\
(1.22,10.8)\end{array}$ & & $\begin{array}{l}\text { HR (Uni- } \\
\text { variate) }\end{array}$ \\
\hline & & & & & & & $\begin{array}{l}p=0.01, \mathrm{HR}=4.69 \\
(1.56,14.2)\end{array}$ & & $\begin{array}{l}\mathrm{HR} \text { (Mul- } \\
\text { tivariate) }\end{array}$ \\
\hline Bowers & 2003 & US & PA141 & $\mathrm{I}$ & $\begin{array}{l}141 \\
(78 / 63)\end{array}$ & $\begin{array}{l}\text { MIB- } \\
1 \geq 2 \%\end{array}$ & & $\begin{array}{l}p=0.02, \mathrm{HR}=1.20 \\
(1.03,1.40)\end{array}$ & $\begin{array}{l}\text { HR (Uni- } \\
\text { variate) }\end{array}$ \\
\hline \multirow{2}{*}{ Zamecnik } & \multirow{2}{*}{2003} & \multirow{2}{*}{$\begin{array}{l}\text { Czech } \\
\text { Re- } \\
\text { public }\end{array}$} & \multirow{2}{*}{ E31 } & \multirow{2}{*}{$\begin{array}{l}\text { II11, III } \\
20\end{array}$} & \multirow{2}{*}{$\begin{array}{l}31 \\
(19 / 12)\end{array}$} & \multirow{2}{*}{$\begin{array}{l}\text { MIB-1 } \\
\text { LI }>7 \%\end{array}$} & $\begin{array}{l}p<0.00, \mathrm{HR}=27.0 \\
(3.5,210.3)\end{array}$ & $\begin{array}{l}p<0.00, \mathrm{HR}=27.3 \\
(3.5,212.3)\end{array}$ & $\begin{array}{l}\text { HR (Uni- } \\
\text { variate) }\end{array}$ \\
\hline & & & & & & & $\begin{array}{l}p=0.03, \mathrm{HR}=8.8 \\
(1.9,48.6)\end{array}$ & $\begin{array}{l}p=0.00, \mathrm{HR}=10.9 \\
(2.3,52.2)\end{array}$ & $\begin{array}{l}\text { HR (Mul- } \\
\text { tivariate) }\end{array}$ \\
\hline Chiang & 2003 & $\begin{array}{l}\text { Tai- } \\
\text { wan }\end{array}$ & $\begin{array}{l}\text { GBM } \\
68\end{array}$ & IV & $\begin{array}{l}68 \\
(41 / 27)\end{array}$ & $\begin{array}{l}\text { MIB- } \\
1>35 \%\end{array}$ & $\begin{array}{l}p<0.01, \mathrm{HR}=2.42 \\
(1.45,4.02)\end{array}$ & & $\begin{array}{l}\text { Survival } \\
\text { curve }\end{array}$ \\
\hline Nede & 2004 & Brazil & $\begin{array}{l}\text { A11, } \\
\text { AA5, } \\
\text { GBM31 }\end{array}$ & & $\begin{array}{l}40 \\
(27 / 13)\end{array}$ & $\begin{array}{l}\text { MIB- } \\
1>3 \%\end{array}$ & $\begin{array}{l}p<0.00 \\
H R=10.241 \\
(2.37,44.26)\end{array}$ & & Estimated \\
\hline Uematsu & 2005 & Japan & ND & $\begin{array}{l}\text { II9, } \\
\text { III12, } \\
\text { IV8 }\end{array}$ & $\begin{array}{l}29 \\
(17 / 12)\end{array}$ & $\begin{array}{l}\mathrm{Ki}-67 \geq \\
10 \%\end{array}$ & \multicolumn{2}{|c|}{$p=0.03, \mathrm{HR}=4.01(1.19,13.5)$} & Estimated \\
\hline Preusser & 2005 & $\begin{array}{l}\text { Aus- } \\
\text { tria }\end{array}$ & E58 & II & 58 & $\begin{array}{l}\mathrm{Ki}-67> \\
20.5 \%\end{array}$ & $\begin{array}{l}p=0.02, \mathrm{HR}=2.42 \\
(1.09,5.40)\end{array}$ & & $\begin{array}{l}\text { Survival } \\
\text { curve }\end{array}$ \\
\hline $\begin{array}{l}\text { Klein- } \\
\text { schmidt- } \\
\text { DeMasters }\end{array}$ & 2006 & US & GBM28 & IV & $\begin{array}{l}28 \\
(22 / 6)\end{array}$ & $\begin{array}{l}\text { MIB-1> } \\
29.3 \%\end{array}$ & \multicolumn{2}{|c|}{$p=0.10, \mathrm{HR}=1.01(0.37,2.75)$} & Estimated \\
\hline Donato & 2007 & Italy & GBM43 & IV & 39 & $\begin{array}{l}\text { MIB- } \\
1>20 \%\end{array}$ & \multicolumn{2}{|c|}{$p=0.00, \mathrm{HR}=4.24(1.68,10.71)$} & $\begin{array}{l}\text { Survival } \\
\text { curve }\end{array}$ \\
\hline $\mathrm{Li}$ & 2009 & China & $\begin{array}{l}\text { primary } \\
\text { GBM } 95 \\
\text { secondary } \\
\text { GBM21 }\end{array}$ & IV & $\begin{array}{l}116 \\
(76 / 40)\end{array}$ & ND & $\begin{array}{l}p=0.009, \mathrm{HR}=2.44 \\
(1.25,4.77)\end{array}$ & $\begin{array}{l}p=0.00, \mathrm{HR}=2.95 \\
(1.47,5.91)\end{array}$ & $\begin{array}{l}\mathrm{HR}(\mathrm{Mul}- \\
\text { tiva } \\
\text { riate) }\end{array}$ \\
\hline Kanamori & 2009 & Japan & $\begin{array}{l}\text { ODG13, } \\
\text { OA5, } \\
\text { AODG } 26, \\
\text { AOA } 12\end{array}$ & II-III & $\begin{array}{l}56 \\
(32 / 24)\end{array}$ & $\begin{array}{l}\mathrm{Ki}-67 \geq \\
25 \%\end{array}$ & & $\begin{array}{l}p=0.00, \mathrm{HR}=3.8 \\
(1.4,9.5)\end{array}$ & $\begin{array}{l}\mathrm{HR} \text { (Mul- } \\
\text { tivariate) }\end{array}$ \\
\hline Laks & 2009 & US & $\mathrm{ND}$ & ND & 30 & ND & $\begin{array}{l}p=0.04, \mathrm{HR}=17.0 \\
(1.0,286)\end{array}$ & $\begin{array}{l}p=0.03, \mathrm{HR}=4.71 \\
(1.12,19.7)\end{array}$ & $\begin{array}{l}\text { HR (Mul- } \\
\text { tiva } \\
\text { riate) }\end{array}$ \\
\hline \multirow{2}{*}{ Kuo } & \multirow{2}{*}{2009} & \multirow{2}{*}{$\begin{array}{l}\text { Tai- } \\
\text { wan }\end{array}$} & \multirow{2}{*}{$\begin{array}{l}\text { ODG } 40 \\
\text { OA9 }\end{array}$} & \multirow{2}{*}{ III } & & Ki-67 & $\begin{array}{l}p=0.05, \mathrm{HR}=2.92 \\
(0.99,8.55)\end{array}$ & $\begin{array}{l}p=0.01, \mathrm{HR}=3.55 \\
(1.36,9.26)\end{array}$ & $\begin{array}{l}\text { HR (Uni- } \\
\text { variate) }\end{array}$ \\
\hline & & & & & $(27 / 22)$ & $\geq 5 \%$ & $p=0.00, \mathrm{HR}=10$ & ,41.67) & $\begin{array}{l}\text { HR (Mul- } \\
\text { tivariate) }\end{array}$ \\
\hline Yoshida & 2009 & Japan & GBM38 & IV & $\begin{array}{l}38 \\
(23 / 15)\end{array}$ & $\begin{array}{l}\text { MIB- } \\
1>20 \%\end{array}$ & $\begin{array}{l}p=0.02, \mathrm{HR}=2.35 \\
(1.04,5.3)\end{array}$ & & $\begin{array}{l}\text { Survival } \\
\text { curve }\end{array}$ \\
\hline Watanabe & 2010 & US & $\begin{array}{l}\text { DA12, } \\
\text { AA9, } \\
\text { GBM44 }\end{array}$ & II-IV & $\begin{array}{l}65 \\
(40 / 25)\end{array}$ & $\begin{array}{l}\text { MIB- } \\
1>25 \%\end{array}$ & $\begin{array}{l}p=0.04, \mathrm{HR}=2.49 \\
(1.03,6.06)\end{array}$ & & $\begin{array}{l}\mathrm{HR} \text { (Mul- } \\
\text { tivariate) }\end{array}$ \\
\hline $\begin{array}{l}\text { Arm- } \\
\text { strong }\end{array}$ & 2010 & US & E63 & II & 63 & $\begin{array}{l}\text { MIB- } \\
1 \geq 10 \%\end{array}$ & $\begin{array}{l}p=0.28, \mathrm{HR}=3.50 \\
(1.15,10.64)\end{array}$ & $\begin{array}{l}p=0.00, \mathrm{HR}=4.69 \\
(1.56,14.2)\end{array}$ & $\begin{array}{l}\text { HR (Uni- } \\
\text { variate) }\end{array}$ \\
\hline
\end{tabular}


DOI:http://dx.doi.org/10.7314/APJCP.2015.16.2.411

Ki-67 is a Valuable Prognostic Factor of Glioma: Evidence from a Systematic Review and Meta-analysis

\begin{tabular}{|c|c|c|c|c|c|c|c|c|c|}
\hline Nabika & 2010 & Japan & $\begin{array}{l}\text { AA24, } \\
\text { GBM35 }\end{array}$ & III-IV & $\begin{array}{l}59 \\
(35 / \\
24)\end{array}$ & $\begin{array}{l}\text { MIB-1> } \\
17.3 \%\end{array}$ & $\begin{array}{l}p=0.31, \mathrm{HR}=1.02 \\
(0.98,1.04)\end{array}$ & & $\begin{array}{l}\text { HR (Mul- } \\
\text { tivariate) }\end{array}$ \\
\hline Zawrocki & 2011 & $\begin{array}{l}\text { Po- } \\
\text { land }\end{array}$ & E39 & II & \begin{tabular}{|l|}
39 \\
$(22 / 17)$ \\
\end{tabular} & $\begin{array}{l}\mathrm{Ki}-67 \\
>3 \%\end{array}$ & $\begin{array}{l}p=0.00, \mathrm{HR}=3.79 \\
(1.23,4.03)\end{array}$ & & $\begin{array}{l}\text { HR (Uni- } \\
\text { variate) }\end{array}$ \\
\hline Jin & 2011 & China & GBM & IV & 156 & $\begin{array}{l}\mathrm{Ki}-67 \\
>25 \% \\
\end{array}$ & $\begin{array}{l}p=0.00, \mathrm{HR}=1.49 \\
(1.06,2.11)\end{array}$ & $\begin{array}{l}p=0.01, \mathrm{HR}=1.16 \\
(1.13,2.18)\end{array}$ & $\begin{array}{l}\text { Survival } \\
\text { curve }\end{array}$ \\
\hline $\begin{array}{l}\text { Habber- } \\
\text { stad }\end{array}$ & 2011 & $\begin{array}{l}\text { Nor- } \\
\text { way }\end{array}$ & AA & III & $\begin{array}{l}27 \\
(17 / 10)\end{array}$ & $\begin{array}{l}\text { MIB- } \\
1>10 \%\end{array}$ & \multicolumn{2}{|c|}{$p=0.03, \mathrm{HR}=2.85(1.03,7.88)$} & Estimated \\
\hline Shen & 2011 & China & $\begin{array}{l}\text { LGA27, } \\
\text { AA20, } \\
\text { GBM24 }\end{array}$ & II-IV & \begin{tabular}{|l|}
71 \\
$(39 / 32)$
\end{tabular} & $\begin{array}{l}\mathrm{Ki}-67 \\
\geq 10 \%\end{array}$ & \multicolumn{2}{|c|}{$p=0.02, \mathrm{HR}=6.45(2.05,16.7)$} & $\begin{array}{l}\text { HR (Mul- } \\
\text { tivariate) }\end{array}$ \\
\hline Margraf & 2011 & US & PA80 & II & \begin{tabular}{|l|}
80 \\
$(40 / 40)$
\end{tabular} & $\begin{array}{l}\text { MIB- } 1 \geq \\
2 \%\end{array}$ & \multicolumn{2}{|c|}{$p=0.03, \mathrm{HR}=1.92(1.04,3.53)$} & $\begin{array}{l}\text { Survival } \\
\text { curve }\end{array}$ \\
\hline Tove & 2012 & $\begin{array}{l}\text { Nor- } \\
\text { way }\end{array}$ & A104 & ND & 104 & $\begin{array}{l}\text { Ki-67> } \\
4.45 \%\end{array}$ & \multicolumn{2}{|c|}{$p=0.26, \mathrm{HR}=1.35(0.70,2.62)$} & $\begin{array}{l}\text { Survival } \\
\text { curve }\end{array}$ \\
\hline \multirow{2}{*}{ Okita } & \multirow{2}{*}{2012} & \multirow{2}{*}{ Japan } & \multirow{2}{*}{ GBM 189} & \multirow{2}{*}{ IV } & \multirow{2}{*}{$\begin{array}{l}189 \\
(121 \\
/ 68)\end{array}$} & $\begin{array}{l}\text { MIB-1> } \\
30 \%\end{array}$ & $\begin{array}{l}p=0.9, \mathrm{HR}=1.07 \\
(0.40,2.93)\end{array}$ & & $\begin{array}{l}\text { HR (Mul- } \\
\text { tivariate) }\end{array}$ \\
\hline & & & & & & $\begin{array}{l}\text { MIB-1> } \\
10 \% \\
\end{array}$ & $\begin{array}{l}p=0.00, \mathrm{HR}= \\
5.25(1.67,20.6)\end{array}$ & & $\begin{array}{l}\mathrm{HR}(\mathrm{Mul} \\
\text { tivariate) }\end{array}$ \\
\hline \multirow{3}{*}{ Yang } & \multirow{3}{*}{2012} & \multirow{3}{*}{ China } & $\mathrm{A}, \mathrm{OA}$ & II & 341 & $\begin{array}{l}\mathrm{Ki}-67 \\
\geq 10 \%\end{array}$ & $\begin{array}{l}p=0.00, \mathrm{HR}=3.40 \\
(1.64,7.02)\end{array}$ & $\begin{array}{l}p=0.09, \mathrm{HR}=1.61 \\
(0.93,2.77)\end{array}$ & $\begin{array}{l}\text { HR (Mul- } \\
\text { tivariate) }\end{array}$ \\
\hline & & & AG & $\mathrm{I}$ & 122 & $\begin{array}{l}\text { ki-67 } \\
\geq 10 \%\end{array}$ & $\begin{array}{l}p=0.72, \mathrm{HR}=0.91 \\
(0.53,1.55)\end{array}$ & $\begin{array}{l}p=0.74, \mathrm{HR}=0.93 \\
(0.62,1.41)\end{array}$ & $\begin{array}{l}\text { HR (Mul- } \\
\text { tivariate) }\end{array}$ \\
\hline & & & GBM & IV & 202 & $\begin{array}{l}\text { ki-67 } \\
\geq 10 \%\end{array}$ & $\begin{array}{l}p=0.49, \mathrm{HR}=1.09 \\
(0.85,1.39)\end{array}$ & $\begin{array}{l}p=0.04, \mathrm{HR}=1.25 \\
(1.01,1.54)\end{array}$ & $\begin{array}{l}\text { HR (Mul- } \\
\text { tivariate) }\end{array}$ \\
\hline Phi & 2012 & Korea & E & II & \begin{tabular}{|l|}
33 \\
$(18 / 15)$ \\
\end{tabular} & \begin{tabular}{|l} 
Ki-67> \\
$8.8 \%$
\end{tabular} & $\begin{array}{l}p=0.43, \mathrm{HR}=1.04 \\
(0.95,1.13)\end{array}$ & $\begin{array}{l}p=0.00, \mathrm{HR}=1.06 \\
(1.02,1.11)\end{array}$ & $\begin{array}{l}\text { HR (Uni- } \\
\text { variate) }\end{array}$ \\
\hline Preusser & 2012 & $\begin{array}{l}\text { Aus- } \\
\text { tria }\end{array}$ & $\mathrm{AOA}$ & III & 76 & $\begin{array}{l}\text { Ki- } 67 \geq \\
22.7 \%\end{array}$ & $\begin{array}{l}p=0.00, \mathrm{HR}=1.92 \\
(1.07,3.46)\end{array}$ & $\begin{array}{l}p=0.00, \mathrm{HR}=2.03 \\
(1.26,3.29)\end{array}$ & $\begin{array}{l}\text { Survival } \\
\text { curve }\end{array}$ \\
\hline \multirow{4}{*}{ Park } & \multirow{4}{*}{2012} & \multirow{4}{*}{ Korea } & \multirow{4}{*}{$\begin{array}{l}\text { LGA } \\
\text { (Biopsy) }\end{array}$} & \multirow{4}{*}{ II } & \multirow{4}{*}{\begin{tabular}{|l|}
33 \\
$(20 / 13)$
\end{tabular}} & \multirow{4}{*}{$\begin{array}{l}\text { MIB- } \\
1>6 \%\end{array}$} & & $\begin{array}{l}p=0.11, \mathrm{HR}=114 \\
(0.97,1.35)\end{array}$ & $\begin{array}{l}\text { HR (Uni- } \\
\text { variate) }\end{array}$ \\
\hline & & & & & & & & $\begin{array}{l}p=0.11, \mathrm{HR}=1.26 \\
(0.95,1.66)\end{array}$ & $\begin{array}{l}\text { HR (Mul- } \\
\text { tivariate) }\end{array}$ \\
\hline & & & & & & & & $\begin{array}{l}p=0.00, \mathrm{HR}=1.17 \\
(1.06,1.29)\end{array}$ & $\begin{array}{l}\text { HR (Uni- } \\
\text { variate) }\end{array}$ \\
\hline & & & & & & & & $\begin{array}{l}p=0.01, \mathrm{HR}=1.25 \\
(1.04,1.50)\end{array}$ & $\begin{array}{l}\text { HR (Mul- } \\
\text { tivariate) }\end{array}$ \\
\hline Liu & 2013 & China & AA & III & \begin{tabular}{|l|}
48 \\
$(31 / 17)$ \\
\end{tabular} & ND & $\begin{array}{l}p<0.01, \mathrm{HR}=4.14 \\
(1.83,9.41)\end{array}$ & $\begin{array}{l}p<0.01, \mathrm{HR}=3.34 \\
(1.53,7.29)\end{array}$ & ND \\
\hline $\begin{array}{l}\text { Abd El } \\
\text { Atti }\end{array}$ & 2013 & Egypt & A111 & ND & \begin{tabular}{|l|}
111 \\
$(74 / 37)$ \\
\end{tabular} & $\begin{array}{l}\text { MIB-1> } \\
10.1 \% \\
\end{array}$ & \multicolumn{2}{|c|}{$p=0.08, \mathrm{HR}=2.66(0.90,7.87)$} & $\begin{array}{l}\text { HR (Mul- } \\
\text { tivariate) }\end{array}$ \\
\hline Huang & 2013 & China & $\begin{array}{l}\text { 41DA, } \\
\text { 30AA, } \\
31 \mathrm{GBM}\end{array}$ & $\begin{array}{l}\text { II41, } \\
\text { III30, } \\
\text { IV31 } \\
\end{array}$ & $\begin{array}{l}102 \\
(55 / 47)\end{array}$ & $\begin{array}{l}\text { Ki-67 } \\
\geq 10 \%\end{array}$ & \multicolumn{2}{|c|}{$p=0.02, \mathrm{HR}=7.75(2.63,18.4)$} & $\begin{array}{l}\text { HR (Mul- } \\
\text { tivariate) }\end{array}$ \\
\hline \multirow[b]{2}{*}{ Tian } & \multirow[b]{2}{*}{2014} & \multirow[b]{2}{*}{ China } & \multirow[b]{2}{*}{ ND } & I-III117 & \multirow{2}{*}{$\begin{array}{l}312 \\
(182 / \\
130)\end{array}$} & \multirow[b]{2}{*}{ ND } & \multirow{2}{*}{\multicolumn{2}{|c|}{$p=0.03, \mathrm{HR}=1.23(0.83,1.61)$}} & \multirow[b]{2}{*}{ ND } \\
\hline & & & & \begin{tabular}{|l} 
III- \\
IV195 \\
\end{tabular} & & & & & \\
\hline \multirow{3}{*}{ Yue } & & & & & & & $\begin{array}{l}p=0.08, \mathrm{HR}=0.62 \\
(0.36,1.06)\end{array}$ & $\begin{array}{l}p=0.38, \mathrm{HR}=1.26 \\
(0.75,2.17)\end{array}$ & $\begin{array}{l}\text { HR (Uni- } \\
\text { variate) }\end{array}$ \\
\hline & 2014 & China & GBM & IV & $\begin{array}{l}62 \\
(43 / 19)\end{array}$ & $\begin{array}{l}\text { Ki-67> } \\
20 \%\end{array}$ & $p=0.09, \mathrm{HR}=1.70$ & $.92,3.13)$ & $\begin{array}{l}\text { HR (Mul- } \\
\text { tivariate) }\end{array}$ \\
\hline & & & & & & & $p=0.02, \mathrm{HR}=1.92$ & $.10,3.33)$ & $\begin{array}{l}\text { HR (Mul- } \\
\text { tivariate) }\end{array}$ \\
\hline
\end{tabular}

*A for astrocytoma;AA for anaplastic astrocytoma;AE for anaplastic ependynoma;AOA for anaplastic oligoastrocytoma;AODG for anaplastic oligodendroglioma;DA for difuse astrocytoma;E for ependynoma;FA for fibrillary astrocytoma; GBM for glioblastoma ;LGA for low grade astrotytoma;ME for myxopapillary ependynoma;OA fo oligoastrocytoma;ODG for oligodendroglioma;PA for pilocytic asrocytoma 
Wen-Jie Chen et al

Table 2. Summarized HRs of Overall and Subgroup Analyses for OS

\begin{tabular}{|c|c|c|c|c|c|}
\hline \multirow[t]{2}{*}{ Stratified analysis } & \multirow[t]{2}{*}{ Study (N) } & \multirow[t]{2}{*}{$\mathrm{HR}$} & \multirow[t]{2}{*}{$p$} & \multicolumn{2}{|c|}{ Heterogeneity } \\
\hline & & & & $\mathrm{I}^{2}$ & $p$ \\
\hline \multicolumn{6}{|l|}{ Region } \\
\hline Europe & 17 & $1.68(1.49,1.90)$ & 0 & $85.30 \%$ & 0 \\
\hline America & 12 & $2.79(1.80,4.33)$ & 0 & $72.10 \%$ & 0 \\
\hline Asia & 16 & $1.73(1.44,2.07)$ & 0 & $85.40 \%$ & 0 \\
\hline Africa & 1 & $2.66(0.90,7.87)$ & 0.08 & - & - \\
\hline \multicolumn{6}{|l|}{ Cutoff } \\
\hline $\mathrm{L}(<10 \%)$ & 18 & $2.42(1.98,2.95)$ & 0 & $88.70 \%$ & 0 \\
\hline $\mathrm{H}(\geq 10 \%)$ & 24 & $1.49(1.34,1.67)$ & 0 & $79.30 \%$ & 0 \\
\hline \multicolumn{6}{|l|}{ Tumor type } \\
\hline A & 29 & $1.73(1.55,1.94)$ & 0 & $84.80 \%$ & 0 \\
\hline OT & 4 & $2.28(1.53,3.40)$ & 0 & $52.20 \%$ & 0.06 \\
\hline $\mathrm{E}$ & 8 & $4.07(1.97,8.42)$ & 0 & $87.60 \%$ & 0 \\
\hline \multicolumn{6}{|l|}{ Method } \\
\hline Estimated & 7 & $3.39(1.91,6.01)$ & 0 & $46.20 \%$ & 0.08 \\
\hline Survival curve & 14 & $2.34(1.82,3.02)$ & 0 & $63.20 \%$ & 0 \\
\hline HR(univariate) & 9 & $2.50(1.38,4.53)$ & 0 & $84.70 \%$ & 0 \\
\hline HR(multivariate) & 15 & $1.34(1.20,1.52)$ & 0 & $80.90 \%$ & 0 \\
\hline
\end{tabular}

Table 3. Summarized HRs of Overall and Subgroup Analyses for PFS

\begin{tabular}{|c|c|c|c|c|c|}
\hline \multirow[t]{2}{*}{ Stratified analysis } & \multirow[t]{2}{*}{ Study $(\mathrm{N})$} & \multirow[t]{2}{*}{ HR } & \multirow[t]{2}{*}{$p$} & \multicolumn{2}{|c|}{ Heterogeneity } \\
\hline & & & & $\mathrm{I}^{2}$ & $p$ \\
\hline \multicolumn{6}{|l|}{ Region } \\
\hline Europe & 2 & $6.81(1.30,35.7)$ & 0.02 & $78.5 \%$ & 0.01 \\
\hline America & 10 & $2.30(1.68,3.15)$ & 0.00 & $71.3 \%$ & 0.00 \\
\hline Asia & 16 & $1.34(1.18,1.53)$ & 0.00 & $76.8 \%$ & 0.00 \\
\hline \multicolumn{6}{|l|}{ Cutoff } \\
\hline $\mathrm{L}(<10 \%)$ & 18 & $1.71(1.40,2.09)$ & 0.00 & $83.3 \%$ & 0.00 \\
\hline $\mathrm{H}(\geq 10 \%)$ & 9 & $1.46(1.22,1.75)$ & 0.00 & $67.0 \%$ & 0.00 \\
\hline \multicolumn{6}{|l|}{ Tumor type } \\
\hline A & 11 & $1.47(1.29,1.66)$ & 0.00 & $65.8 \%$ & 0.00 \\
\hline OT & 2 & $4.42(2.40,8.15)$ & 0.00 & $0.00 \%$ & 0.46 \\
\hline $\mathrm{E}$ & 5 & $5.83(1.84,18.48)$ & 0.00 & $89.7 \%$ & 0.00 \\
\hline \multicolumn{6}{|l|}{ Method } \\
\hline Estimated & 2 & $3.53(1.70,7.33)$ & 0.00 & $0.00 \%$ & 0.82 \\
\hline Survival curve & 14 & $1.98(1.52,2.59)$ & 0.00 & $55.7 \%$ & 0.02 \\
\hline HR(univariate) & 9 & $1.29(1.09,1.52)$ & 0.00 & $81.9 \%$ & 0.00 \\
\hline HR(multivariate) & 15 & $1.78(1.35,2.33)$ & 0.00 & $72.8 \%$ & 0.00 \\
\hline
\end{tabular}

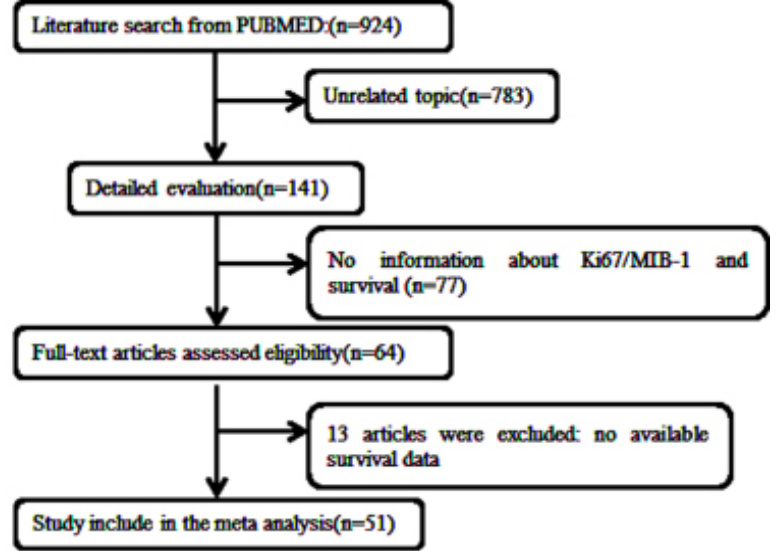

Figure 1. Flow Diagram of Study Selection

oligoastrocytic tumors, 8 studies included ependymomas, 2 studies included several glioma types and 3 studies showed no information about tumor type. Moreover, there were 11 studies in the astrocytoma group, 5 studies in the ependymoma group, 2 studies with oligodendroglial and oligoastrocytic tumors, 1study including several glioma

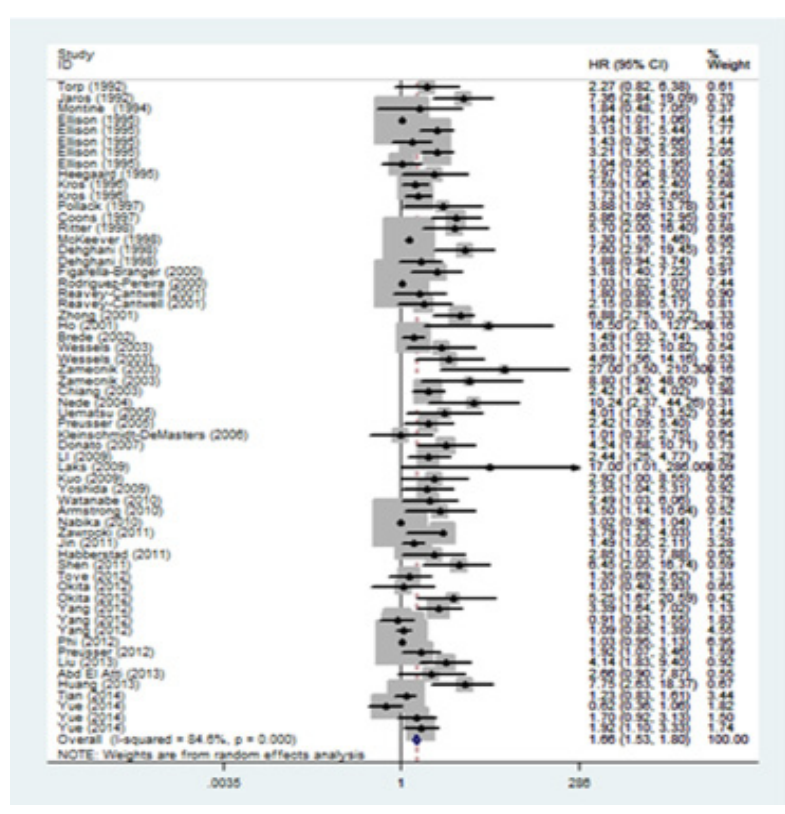

Figure 2. Forest Plot Showing the Combined Relative HR from Random-effect for OS 


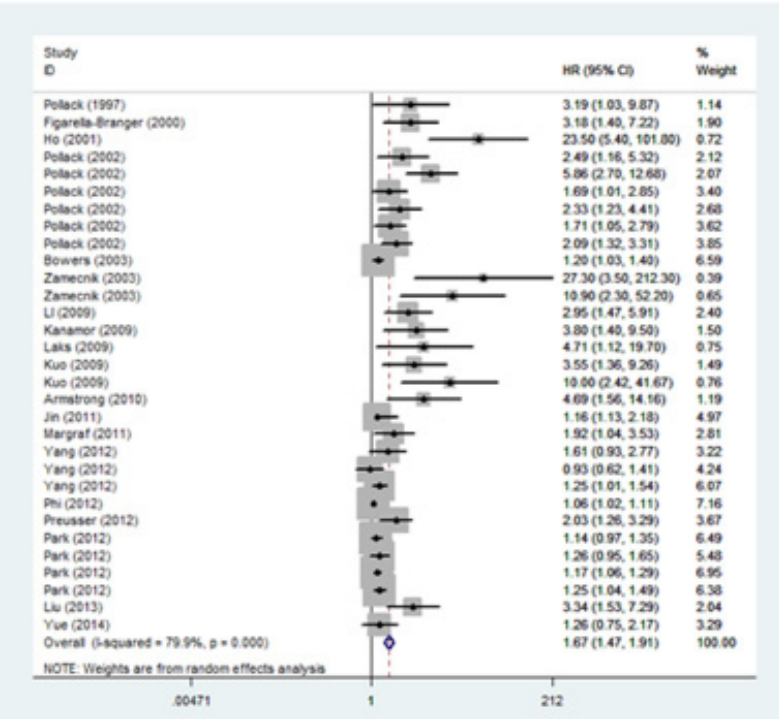

Figure 3. Forest Plot Showing the Combined Relative HR form Random-effect PFS

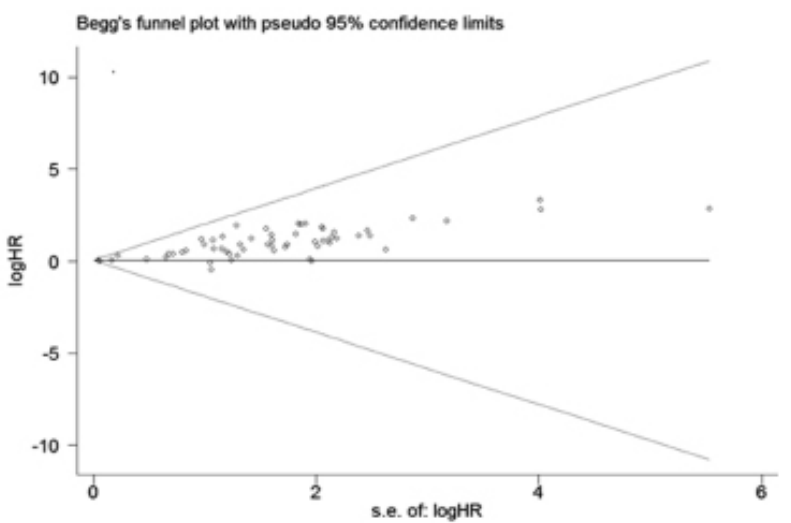

Figure 4. Funnel Blot was Designed to Visualize a Potential Poblication Bias for OS Stidies

types and 1 study with no information about glioma type in PFS analysis group. The cut-offs of antibodies used to define immunohistochemical positivity also varied between reports, 42 OS analysis group selected the percentage of positive staining as the cut-off point, including 24 studies $\geq 10 \%, 18$ studies $<10 \%$.

\section{Meta-analysis}

In this meta-analysis, we evaluated 46 studies dealing with Ki-67 expression and OS. The pooled HR was 1.66 (95\% CI: $1.53-1.80 ; Z=11.87 ; p=0.000$, Figure 2$)$ with Heterogeneity $\left(\mathrm{I}^{2} 84.6 \% p=0.000\right)$. It suggested that overexpression of $\mathrm{Ki}-67$ was significantly related with the worse prognosis of glioma and high Ki-67 expression was a valuable prognostic factor for overall survival in glioma patients. Moreover, we also performed subgroup analysis by region, glioma pathology type, cut-off value and statistical method. The result showed that significant relationships between high Ki-67 expression and OS were exhibited especially in the subgroup of oligodendroglial and oligoastrocytic tumors $(\mathrm{HR}=1.99,95 \% \mathrm{CI}, 1.56,2.55$; $\mathrm{Z}=5.48, p=0.000)$ and HR estimated by original data $(\mathrm{HR}=3.28,95 \%$ 2.16, 4.97; $\mathrm{Z}=5.60, p=0.000$, Table2). In the subgroup analysis according to the study region, the combined HR was 2.79 (95\%CI: 1.80-4.33; $\mathrm{Z}=4.60$;

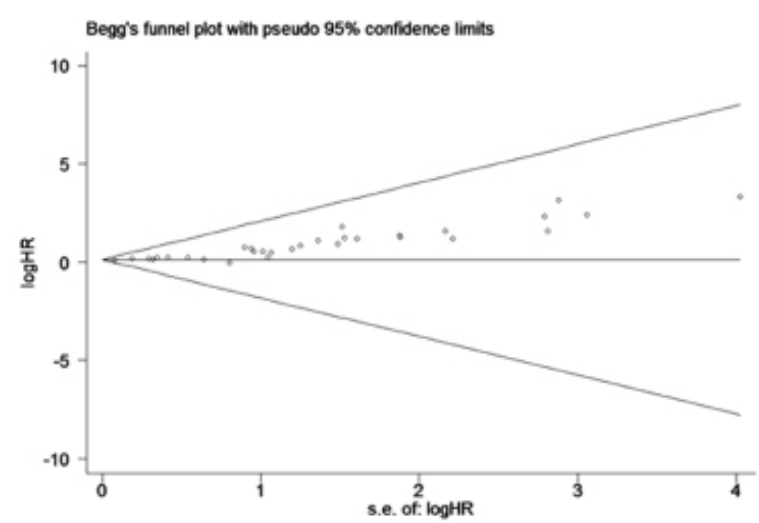

Figure 5. Funnel Blot was Designed to Visualize a Potential Poblication Bias for PFS Stidies

$p=0.000$ ) for America countries. When stratified according to the cut-off value, the combined HRs of the subgroup with cut-off $<10 \%$ showed an inverse effects on survival (HR=2.42; 95\%CI 1.98-2.95; $\mathrm{Z}=8.72, p=0.000$, Table2).

The nineteen studies in PFS analysis group were also statistically significant with a pooled estimate of risk of 1.67 (95\%CI:1.47-1.91; Z=7.67; $p=0.000$, Figure3) with Heterogeneity $\left(\mathrm{I}^{2} 79.9 \% p=0.000\right)$. Statistically significant differences were also identified for all survival subgroup analyses (Table3).

\section{Heterogeneity analysis results}

Obvious heterogeneity of subjects was found in 3 of the 4 analysis groups (OS for all, $p=0.00, \mathrm{I}^{2}=84.6 \%$; OS for astocytoma, $p=0.000$ and $\mathrm{I}^{2}=84.8 \%$; OS for oligodendroglial and oligoastrocytic tumors, $p=0.063$, $\mathrm{I}^{2}=52.2 \%$; OS for ependymoma, $p=0.000, \mathrm{I}^{2}=87.6 \%$ ). Meanwhile, we conducted a meta regression to evaluate the potential factors responsible for the obvious heterogeneity (Table2). In the subgroup that HR estimated by original data did not show obvious heterogeneity $\left(p=0.084, \mathrm{I}^{2}=46.2 \%\right)$. In the PFS analysis group, obvious heterogeneity of subjects was also found (PFS for all, $\left.p=0.000, \mathrm{I}^{2}=79.9 \%\right)$. However, PFS for oligodendroglial and oligoastrocytic tumors $\left(p=0.458, \mathrm{I}^{2}=0.0 \%\right)$ and HR estimated by original data $\left(p=0.861, \mathrm{I}^{2}=0.0 \%\right)$ reached low heterogeneity (Table3).

\section{Publication Bias}

Figure4 and Figure 5 showed that the funnel plot was unsymmetrical, that indicated there existed publication bias. The Begg rank correction test also detected evidence for publication bias among studies of Ki-67 and OS $(p=0.04)$ and PFS $(p<0.001)$ in glioma patients. The funnel plot revealed an apparent asymmetry that suggested the presence of a potential publication bias, a language bias, inflated estimates by a flawed methodologic design in smaller studies, and/or a lack of publication of small trials with opposite results.

\section{Discussion}

This meta-analysis showed that Ki-67 overexpression was associated with worse OS and PFS in glioma patients. Recently, a few studies have suggested that Ki-67 is an essential prognostic factor in human glioma (Yoshida et 
al., 2010; Preusser et al., 2012). In our systematic review with meta-analysis, patients with $\mathrm{Ki}-67$ positive tumors had significantly worse survival than those with Ki-67 negative ones. The mechanism underlying the effect of Ki67 expression on tumor progression and prognosis remains essentially uncertain.However, it has to be considered that positivity for the Ki-67 antigen may reflect the ability of a cell to continue to proliferate after the time of tumor resection.This nonhistone nuclear protein is expressed throughout the active parts of the cell cycle $(\mathrm{G} 1, \mathrm{~S}, \mathrm{G} 2$, and mitosis). The Ki-67 protein can be detected during all active phases of the cell cycle but not in resting cells and can be used as a tool to estimate the growth fraction of any human cell population. Besides, Ki-67 immunostaining can easily be performed on various types of cytological and histological preparations, such as smear, squash, cytocentrifuge preparations, and histological sections. Thus, the importance of the Ki-67/MIB-1 labeling index (LI) as a prognostic and predictive factor in human malignancies has been debated for many years.

In this meta-analysis, we had dealt with highly significant heterogeneity among OS analysis and PFS analysis group. Although we used random effects models to analyze the data, heterogeneity was still apotential problem to affect the results of meta-analysis. The information of the included studies revealed that the heterogeneity could be attributed to the differences in the publication years, the pathology types and stages of the tumors, the cut-off values of Ki-67, study region and the risk evaluation methods.

Our meta-analysis was carried out using literature published results, and we therefore acknowledge some limitations of our approach which is, however, much less expensive than a meta-analysis using individual patients data. The language selection can favour positive studies, following the assumption that they are more often published in English, whereas the negative ones tend to be published more often in local journals.

One of the possible source of confusion is the usage of the same cohort of patients in different publications. It might be difficult to avoid inclusion of some patients more than once in the meta-analysis, although publications clearly based on the analyses of the same patients cohorts were excluded. We have assumed that authors have been honest and have not reported the results from the same cohort of patients without mentioning in their publications.

Another potential source of bias is related to the method for extrapolating the HR. If the authors did not report the individual HR together with its variance, we calculated it from the survival comparison statistic and its variance whenever possible. If not, we extrapolated it from the survival curves using several time points during follow-up for reading the corresponding survival rates, assuming that censored observations were uniformly distributed. Reading the survival rates on the graphical representation of the survival curves was performed independently by three of the authors, but this strategy can not eliminate complete inaccuracy in the extracted survival rates. Consequently, the estimated HR might be less reliable than when obtained from published statistics.

Additionally, some studies have used $10 \%$ as the cut- off (arbitrary value), whereas others have chosen mean, median, the optimal cut-off value or arbitrary values. These differences might be responsible for the difficulty in determining a standard threshold in daily practice. In the context of this meta-analysis, we may assume that increased Ki-67 leads to an increased risk of relapse and/ or death and that a relative increase is estimated although the baseline risk (the risk in the group considered Ki-67 negative) is not the same in all the studies.

In conclusion, this meta-analysis has yielded significant association between $\mathrm{Ki}-67$ overexpression and worse OS and PFS in patients with glioma.Ki-67 can be a potential prognostic indicator for glioma patients. In addition, it is rather necessary that better designed studies need to be enrolled into such kind of analysis in the future, to provide a better conclusion about the relationship between Ki-67 expression and the outcome of patients with glioma. The value of Ki-67 for molecular staging of glioma also needs to be confirmed in controlled trials involving larger number of patients with longer follow-up, before any definitive conclusions can be made.

\section{Acknowledgements}

The study was supported by the fund of Guangxi Zhuang Autonomous Region University Student Innovative Plan (No. 201410598026), China. The funders had no role in study design, data collection and analysis, decision to publish, or preparation of the paper.

\section{References}

Abd El Atti RM, Abou Gabal HH, Osman WM, et al (2013). Insights into the prognostic value of DJ-1 and MIB-1 in astrocytic tumors. Diagn Pathol, 8, 126.

Armstrong TS, Vera-Bolanos E, Bekele BN, et al (2010). Adult ependymal tumors: prognosis and the M.D. anderson cancer center experience. Neuro Oncol, 12, 862-70.

Bowers DC (2003). Study of the MIB-1 labeling index as a predictor of tumor progression in pilocytic astrocytomas in children and adolescents. J Clin Oncol, 21, 2968-73.

Bredel M, Piribauer M, Marosi C, et al (2002). High expression of DNA topoisomerase II $\alpha$ and $\mathrm{Ki}-67$ antigen is associated with prolonged survival in glioblastoma patients. European J Cancer, 38, 1343-7.

Chiang MDH, Ting ML-T, Hsu MDMHAC-Y, et al (2003). MIB1 and DNA topoisomerase IIa could be helpful for predicting long-term survival of patients with glioblastoma. Am J Clin Pathol, 119, 715-22.

Coons SW, Johnson PC, Pearl DK (1997). The prognostic significance of Ki-67 labeling indices for oligodendrogliomas. Neurosurgery, 41, 878-85.

Dang YW, Zeng J, He RQ, et al (2014). Effects of miR-152 on cell growth inhibition, motility suppression and apoptosis induction in hepatocellular carcinoma cells. Asian Pac J Cancer Prev, 15, 4969-76.

Dehghani F, Schachenmayr W, Laun A, et al (1998). Prognostic implication of histopathological, immunohistochemical and clinical features of oligodendrogliomas: a study of 89 cases. Acta Neuropathologica, 95, 493-504.

Donato V, Papaleo A, Castrichino A, et al (2007). Prognostic implication of clinical and pathologic features in patients with glioblastoma multiforme treated with concomitant radiation plus temozolomide. Tumori, 93, 248. 
Ki-67 is a Valuable Prognostic Factor of Glioma: Evidence from a Systematic Review and Meta-analysis

Ellison DW, Steart PV, Bateman AC, et al (1995). Prognostic indicators in a range of astrocytic tumours: an immunohistochemical study with Ki-67 and p53 antibodies. J Neurol Neurosurg Psychiatry, 59, 413-9.

Fakhrjou A, Dastranj-Tabrizi A, Ghojazadeh M, et al (2013). Diagnostic value of protein Ki67 (MIB-1) in atypical Pap smears of postmenopausal women. Asian Pac J Cancer Prev, 14, 4815-8.

Figarella-Branger D, Civatte M, Bouvier-Labit C, et al (2000). Prognostic factors in intracranial ependymomas in children. J Neurosurg, 93, 605-13.

Habberstad AH, Gulati S, Torp SH (2011). Evaluation of the proliferation markers Ki-67/MIB-1, mitosin, survivin, $\mathrm{pHH}$, and DNA topoisomerase IIa in human anaplastic astrocytomas-an immunohistochemical study. Diagn Pathol, 6, 43 .

Haroon S, Hashmi AA, Khurshid A, et al (2013). Ki67 index in breast cancer: correlation with other prognostic markers and potential in pakistani patients. Asian Pac J Cancer Prev, 14, 4353-8.

Heegaard S, Sommer HM, Broholm H, et al (1995). Proliferating cell nuclear antigen and Ki-67 immunohistochemistry of oligodendrogliomas with special reference to prognosis. Cancer, 76, 1809-13.

Hernandez-Pedro NY, Rangel-Lopez E, Magana-Maldonado R, et al (2013). Application of nanoparticles on diagnosis and therapy in gliomas. Biomed Res Int, 2013, 351031.

Higgins J, Thompson SG (2002). Quantifying heterogeneity in a meta-analysis. Statistics in medicine, 21, 1539-58.

Ho DM-T, Hsu C-Y, Wong T-T, et al (2001). A clinicopathologic study of 81 patients with ependymomas and proposal of diagnostic criteria for anaplastic ependymoma. J NeuroOncol, 54, 77-85.

Hu X, Miao W, Zou Y, et al (2013). Expression of p53, epidermal growth factor receptor, Ki-67 and O6-methylguanine-DNA methyltransferase in human gliomas. Oncol Letters, 6, 130-4.

Huang S, Chen G (2014). Overexpression of DcR3 and its significance on tumor cell differentiation and proliferation in glioma. Scientific World J, 2014, 605236.

Huang T, Jin X, He L, et al (2013). Role of podocalyxin in astrocytoma: clinicopathological and evidence. Oncol Lett, 6, 1390-6.

Jaros E, Perry R, Adam L, et al (1992). Prognostic implications of p53 protein, epidermal growth factor receptor, and Ki-67 labelling in brain tumours. Br J Cancer, 66, 373.

Kanamori M, Kumabe T, Sonoda Y, et al (2008). Predictive factors for overall and progression-free survival, and dissemination in oligodendroglial tumors. J Neuro-Oncol, 93, 219-28.

Kleinschmidt-DeMasters BK, Meltesen L, McGavran L, et al (2006). Characterization of glioblastomas in young adults. Brain Pathology, 16, 273-86.

Kohler BA, Ward E, McCarthy BJ, et al (2011). Annual report to the nation on the status of cancer, 1975-2007, featuring tumors of the brain and other nervous system. J Natl Cancer Inst, 103, 714-36

Kouri FM, Jensen SA, Stegh AH (2012). The role of Bcl-2 family proteins in therapy responses of malignant astrocytic gliomas: Bcl2L12 and beyond. Scientific World J, 2012 , 838916.

Kros JM, Hop WC, Godschalk JJ, et al (1996). Prognostic value of the proliferation-related antigen $\mathrm{Ki}-67$ in oligodendrogliomas. Cancer, 78, 1107-13.

Kuo LT, Kuo KT, Lee MJ, et al (2009). Correlation among pathology, genetic and epigenetic profiles, and clinical outcome in oligodendroglial tumors. Int J Cancer, 124, 2872-9.
Laks DR, Masterman-Smith M, Visnyei K, et al (2009). Neurosphere formation is an independent predictor of clinical outcome in malignant glioma. Stem Cells, 27, 980-7.

Lau J, Ioannidis JP, Schmid CH (1997). Quantitative synthesis in systematic reviews. Ann Intl Med, 127, 820-6.

Li S-W, Qiu X-G, Chen B-S, et al (2009). Prognostic factors influencing clinical outcomes of glioblastoma multiforme. Chin Med J, 122, 1245-9.

Liu Y, Tang K, Yan W, et al (2013). Identifying Ki-67 specific miRNA-mRNA interactions in malignant astrocytomas. Neuroscience Letters, 546, 36-41.

Margraf LR, Gargan L, Butt Y, et al (2011). Proliferative and metabolic markers in incompletely excised pediatric pilocytic astrocytomas-an assessment of 3 new variables in predicting clinical outcome. Neuro Oncol, 13, 767-74.

McKeever PE, Strawderman MS, Yamini B, et al (1998). MIB1 proliferation index predicts survival among patients with grade II astrocytoma. J Neuropathol Exp Neurol, 57, 931-6.

Montine TJ, Vandersteenhoven JJ, Aguzzi A, et al (1994). Prognostic significance of Ki-67 proliferation index in supratentorial fibrillary astrocytic neoplasms. Neurosurgery, 34, 674-9.

Nabika S, Kiya K, Satoh H, et al (2010). Prognostic significance of expression patterns of EGFR family, p21 and p27 in high-grade astrocytoma. Hiroshima J Med Sci, 59, 65-70.

Nazarenko I, Hede SM, He X, et al (2012). PDGF and PDGF receptors in glioma. Ups $J$ Med Sci, 117, 99-112.

Neder L, Colli B, Machado H, et al (2003). MIB-1 labeling index in astrocytic tumors-a clinicopathologic study. Clin Neuropathol, 23, 262-70.

Okita Y, Narita Y, Miyakita Y, et al (2012). Pathological findings and prognostic factors in recurrent glioblastomas. Brain Tumor Pathol, 29, 192-200.

Park KJ, Kang SH, Park DH, et al (2012). Usefulness of thallium-201 SPECT for prediction of early progression in low-grade astrocytomas diagnosed by stereotactic biopsy. Clin Neurol Neurosurg, 114, 223-9.

Parmar MK, Torri V, Stewart L (1998). Extracting summary statistics to perform meta-analyses of the published literature for survival endpoints. Stat Med, 17, 2815-34.

Phi JH, Wang KC, Park SH, et al (2012). Pediatric infratentorial ependymoma: prognostic significance of anaplastic histology. J Neurooncol, 106, 619-26.

Pollack IF, Campbell JW, Hamilton RL, et al (1997). Proliferation index as a predictor of prognosis in malignant gliomas of childhood. Cancer, 79, 849-56.

Pollack IF, Hamilton RL, Burnham J, et al (2002). Impact of proliferation index on outcome in childhood malignant gliomas: results in a multi-institutional cohort. Neurosurgery, 50, 1238-45.

Preusser M, Hoeftberger R, Woehrer A, et al (2012). Prognostic value of Ki67 index in anaplastic oligodendroglial tumours-a translational study of the European organization for research and treatment of cancer brain tumor group. Histopathology, 60, 885-94.

Preusser M, Wolfsberger S, Czech T, et al (2005). Survivin expression in intracranial ependymomas and its correlation with tumor cell proliferation and patient outcome. Am J Clin Pathol, 124, 543-9.

Qiang J, Wei Z, Xiao-Guang Q, et al (2011). Gene expression profiling reveals $\mathrm{Ki}-67$ associated proliferation signature in human glioblastoma. Chinese Med J Beijing, 124, 2584.

Reavey-Cantwell JF, Haroun RI, Zahurak M, et al (2001). The prognostic value of tumor markers in patients with glioblastoma multiforme: analysis of 32 patients and review of the literature. J Neuro-Oncol, 55, 195-204.

Ritter AM, Hess KR, McLendon RE, et al (1998). Ependymomas: 
MIB-1 proliferation index and survival. J Neurooncol, 40, 51-7.

Rodriguez-Pereira C, Suarez-Penaranda J, Vazquez-Salvado $\mathrm{M}$, et al (2000). Value of MIB-1 labelling index (LI) in gliomas and its correlation with other prognostic factors. A clinicopathologic study. J Neurosurg Sci, 44, 203-9; discussion 9-10.

Shen C, Yang L, Yuan X (2011). Endothelin B receptor expression in human astrocytoma: association with clinicopathological variables and survival outcomes. Int J Neurosci, 121, 626-31.

Tadbir AA, Pardis S, Ashkavandi ZJ, et al (2012). Expression of Ki67 and CD105 as proliferation and angiogenesis markers in salivary gland tumors. Asian Pac J Cancer Prev, 13, 5155-9.

Tian B, Zhang Y, Zhang J (2014). Periostin is a new potential prognostic biomarker for glioma. Tumour Biol, 35, 5877-83.

Torp S, Helseth E, Dalen A, et al (1992). Relationships between Ki-67 labelling index, amplification of the epidermal growth factor receptor gene, and prognosis in human glioblastomas. Acta Neurochirurgica, 117, 182-6.

Tove L-L, Hanssøn HA, Stein S, et al (2012). Prognostic value of histological features in diffuse astrocytomas WHO grade II. Interl J Clin Experimental Pathol, 5, 152.

Uematsu M, Ohsawa I, Aokage T, et al (2005). Prognostic significance of the immunohistochemical index of survivin in glioma: a comparative study with the MIB-1 index. $J$ Neurooncol, 72, 231-8.

Watanabe Y, Yamasaki F, Kajiwara Y, et al (2010). Expression of phosphoprotein enriched in astrocytes $15 \mathrm{kDa}$ (PEA$15)$ in astrocytic tumors: a novel approach of correlating malignancy grade and prognosis. J Neurooncol, 100, 449-57.

Wen PY, Kesari S (2008). Malignant gliomas in adults. New England J Med, 359, 492-507.

Wessels PH, Hopman AH, Kubat B, et al (2003). Proliferation and aneusomy predict survival of young patients with astrocytoma grade II. Br J Cancer, 89, 128-34.

Westermark B (2012). Glioblastoma-a moving target. Ups $J$ Med Sci, 117, 251-6.

Yang P, Wang Y, Peng X, et al (2013). Management and survival rates in patients with glioma in China (2004-2010): a retrospective study from a single-institution. J Neurooncol, 113, 259-66.

Yoshida Y, Nakada M, Harada T, et al (2010). The expression level of sphingosine-1-phosphate receptor type 1 is related to MIB-1 labeling index and predicts survival of glioblastoma patients. J Neurooncol, 98, 41-7.

Yue Q, Zhang X, Ye HX, et al (2014). The prognostic value of Foxp3+ tumor-infiltrating lymphocytes in patients with glioblastoma. J Neurooncol, 116, 251-9.

Zamecnik J, Snuderl M, Eckschlager T, et al (2003). Pediatric intracranial ependymomas: prognostic relevance of histological, immunohistochemical, and flow cytometric factors. Modern Pathol, 16, 980-91.

Zawrocki A, Izycka-Swieszewska E, Papierz W, et al (2011). Analysis of the prognostic significance of selected morphological and immunohistochemical markers in ependymomas, with literature review. Folia Neuropathol, 49, 94-102.

Zhao S, Wu J, Wang C, et al (2013). Intraoperative fluorescenceguided resection of high-grade malignant gliomas using 5-aminolevulinic acid-induced porphyrins: a systematic review and meta-analysis of prospective studies. PLoS One, 8, e63682.

Zhong D, Li X, Zhang G (2001). Multivariate analysis of the parameters related to prognosis of astrocytoma. Zhonghua Bing Li Xue Za Zhi Chinese J Pathol, 30, 345-9. 